Trayectorias digitales universitarias. Aproximaciones a un campo en construcción

Melina Milagros Pereyra

Question/Cuestión, Nro.70, Vol.3, diciembre 2021

ISSN: 1669-6581

URL de la Revista: https://perio.unlp.edu.ar/ojs/index.php/question/

IICom -FPyCS -UNLP

DOI: $\underline{\text { https//doi.org/10.24215/16696581e592 }}$

\title{
Trayectorias digitales universitarias
}

\section{Aproximaciones a un campo en construcción}

\section{University digital trajectories}

\section{Approaches to a field under construction}

Melina Milagros Pereyra

Instituto de Investigaciones en Comunicación (IICOM) Facultad de Periodismo y Comunicación Social (FPyCS) Universidad Nacional de La Plata

Argentina

https://orcid.org/0000-0002-2891-6710

pereyramel.99@gmail.com

\section{Resumen}

El presente artículo pone en común los aportes de diversos autoras y autores que ayudaron a la construcción y delimitación del tema/problema en el proyecto de investigación de 
la beca de Estímulo a las Vocaciones Científicas del Consejo Interuniversitario Nacional (EVCCIN) (2021/22) 'Trayectorias estudiantiles digitales universitarias: las experiencias educativas de las y los estudiantes de la carrera de comunicación en la FPyCS-UNLP'.

El mismo se pregunta por las trayectorias educativas en tanto su incidencia el acceso, uso y apropiación de dispositivos tecnológicos y de conectividad en un contexto de pandemia/post pandemia tomando como caso de estudio las experiencias de las y los jóvenes estudiantes del ciclo básico de la Licenciatura en Comunicación Social de la Facultad de Periodismo y Comunicación Social de la UNLP.

Por consiguiente, se toman y se ponen en diálogo las producciones científicoacadémicas que han tenido lugar en el plano nacional y regional durante los últimos 15 años en torno a la temática que atraviesa al tema que se investiga en dicha beca.

Palabras clave: trayectorias educativas; TICs; juventudes; pandemia

\section{Abstract}

This article brings together the contributions of various authors who helped in the construction and delimitation of the topic/problem of the EVC-CIN Grant Research Project "Educational and digital trajectories at University: The educational experiences of communication students at the FPyCS-UNLP". It asks about educational trajectories in terms of the impact that access, use and appropriation of technological and connectivity devices have on them in a context of pandemic/post pandemic, taking as a case study the experiences of young students of the basic cycle of the Bachelor's Degree in Social Communication of the School of Journalism and Social Communication of the UNLP. Therefore, the scientific-academic productions that have taken place at the national and regional level during the last 15 years on the subject that crosses this scholarship are taken and put in dialogue. 
Key words:educational trajectories; ICTs; younghood; pandemic

\section{A modo de introducción}

El presente artículo se enmarca en el Proyecto de Beca de Estímulo a las Vocaciones Científicas del Consejo Interuniversitario Nacional ( $\mathrm{CiN}$ ) titulado "Trayectorias estudiantiles digitales universitarias: las experiencias educativas de las y los estudiantes de la carrera de comunicación en la FPyCS-UNLP" que se desarrolla durante el período septiembre 2021-2022.

Aquí, la pregunta central es por las trayectorias estudiantiles digitales universitarias de las y los estudiantes del ciclo básico de la Licenciatura de Comunicación Social en la Facultad de Periodismo y Comunicación de la Universidad Nacional de La Plata en un momento histórico de emergencia sanitaria debido a la pandemia por COVID-19.

A partir del reconocimiento de un lugar de vacancia en el estudio de las trayectorias educativas situadas en la convergencia digital, este proyecto propone una indagación en los modos en que las y los estudiantes acceden, usan y se apropian de las tecnologías de la información y la comunicación (TICs), y cómo inciden en su recorrido por la Universidad.

Asimismo, se pretende categorizar cómo se configuran estas trayectorias en términos de hacer una diferenciación entre "ideales" y "reales". Conocer sobre la construcción del oficio de estudiante universitario y dar cuenta de las tensiones existentes entre las actividades académicas (cursadas, estudio) y las extracadémicas (trabajo, deporte, hobbies, militancia) resulta fundamental para comprender en profundidad la trayectoria de cada estudiante de dicha Casa de Estudios.

De modo que, la hipótesis de este plan de trabajo plantea que las trayectorias digitales de los y las estudiantes de la FPyCS son diversas, heterogéneas y están atravesadas por sus actividades extracurriculares y el acceso real a dispositivos tecnológicos y de conectividad. Por lo que se generan tensiones entre sus trayectorias reales e ideales, frente a lo cual la Unidad Académica emplea dispositivos de acompañamiento para garantizar la continuidad. $Y$ en este marco van definiendo su oficio de estudiantes universitarios/as. 
En el campo académico-científico es importante pensar en el futuro que vendrá algunos/as ya dirán "post pandémico", otros/as pensarán aun "pandémico"- desde una perspectiva que se preocupe e indague en los procesos de acceso, uso, apropiación y circulación de los procesos socioculturales que hace cada sujeto, cada estudiante universitaria/o. Un porvenir que ponga el foco en las trayectorias estudiantiles digitales universitarias es sin dudas un mañana por construir.

\section{Configuración de trayectorias: estudiante ideal versus estudiante real}

Se parte del concepto de trayectorias educativas para analizar las particularidades y diferencias que tiene el recorrido que hace cada estudiante por la Universidad al tiempo que se pone en discusión la expectativa institucional en torno a las mismas, diferenciando al estudiante "ideal" del estudiante "real".

Claudia Bracchi (2016) es una autora clave para problematizar el concepto. Ella plantea que las trayectorias son experiencias vitales y heterogéneas que permiten reconocer los ámbitos formativos por los que transcurre el estudiantado mientras va conformando su biografía (experiencias laborales, militancia política, primer trabajo, actividades extracurriculares). Es decir, todos aquellos condicionantes (experiencias, saberes, etc) que interpelan y moldean al sujeto en su camino por la institución educativa.

Aquí, la autora argentina retoma el concepto de capital cultural de Pierre Bourdieu (1979), entendido como acumulación no solo de bienes materiales sino también de conocimiento, ciencia, y arte, el cual se impone como un punto para explicitar las desigualdades en las experiencias educativas por lo que tendrá un rol definitorio en la construcción de la trayectoria estudiantil.

El ingreso de los estudiantes a una carrera universitaria implica un cambio que no es solamente en términos institucionales o curriculares sino se ponen en juego los esquemas de percepción, de apreciación, valorización y de acción (habitus) que harán posible diversas toma de decisiones contribuyendo a conformar el oficio de estudiante universitario. Dicho oficio se conforma apropiándose de las reglas de juego de la institución y para ello deberá utilizar todo 
su capital económico, cultural y simbólico. En este marco cobran importancia las estrategias que los estudiante estén en condiciones de elaborar y desarrollar (p.5)

Es interesante resaltar que Bracchi en la Conferencia para FILO UBA (2020) piensa a las trayectorias desde tres dimensiones: las condiciones materiales/objetivas, esto es, los indicadores socioeconómicos y culturales y en en la pregunta que esta investigación se hace, en el acceso a conectividad y dispositivos tecnológicos; los aspectos o condicionantes simbólicos-subjetivos, las formas de pensar, identificaciones y significaciones sociales; y los aspectos institucionales en tanto se sitúa el lugar del proyecto político con las políticas educativas que crean las condiciones para el desarrollo de las trayectorias.

En este contexto las y los estudiantes van definiendo su oficio de estudiantes universitarios entendido como «los modos (estrategias, resistencias, formas de relacionarse, la participación estudiantil) por los cuales se va aprendiendo a ser estudiante universitario» (Bracchi, 2016, p.8). Además, en este "aprender a ser", el o la estudiante debe incorporar las lógicas propias de la Universidad y facultad en la que se inserta, un tiempo escolar nuevo, el trabajo pedagógico, otras formas de aprendizaje y conocimiento, vinculación con sus pares (compañeros/as) y con el equipo docente.

Hay tres etapas que marcan el pasaje de la escuela secundaria a la Universidad. En primer lugar, el tiempo de extrañamiento, cuando el/la estudiante entra por primera vez a una institución desconocida. En segundo lugar, el tiempo de aprendizaje, mientras el/la estudiante va adquiriendo y adaptándose a las reglas institucionales. Y tercero, el tiempo de afiliación, donde el/la estudiante domina dicha reglas lo cual implica un nuevo manejo del tiempo, de la autonomía y de las condiciones que estimulan a generar sus propias prácticas (Coulon, 1995, como se citó en Bracchi, 2016).

Dado que este proyecto de beca toma como caso de estudio a jóvenes estudiantes de la FPyCS, el concepto de condición juvenil es sustancial para pensar y categorizar de manera heterogénea y diversa el camino que hacen por esta Facultad. Rossana Reguillo (2013) entiende que «los jóvenes no constituyen una categoría homogénea, no comparten los modos de inserción en la estructura social, lo cual implica una cuestión de fondo: sus esquemas de representación configuran campos de acción diferenciados y desiguales» (p.26). 
En este sentido, se reconocen dos tipos de actores juveniles según la vinculación de las y los jóvenes con la estructura o sistema en el que se ubican: los "incorporados" cuyas prácticas son analizadas desde el ámbito escolar, laboral, religioso o consumo cultural; y los "alternativos" o "disidentes", los cuales son estudiados desde su no-incorporación a los esquemas de la cultura dominante (Reguillo, 2013).

Además, la investigadora mexicana hace hincapié en que la definición de juventud(es) no puede reducirse únicamente a un determinado rango etario al tiempo que pone en valor de la historización, es decir, el ponerlas siempre en contexto con el momento social, político, educativo, económico y cultural en el que están inmersas: Los jóvenes, desde esta mirada, son vistos como sujetos de discurso y como agentes sociales, con capacidad para apropiarse de (y movilizar) los objetos tanto sociales y simbólicos como materiales. En otras palabras, se les reconoce el papel activo en su capacidad de negociación con las instituciones y estructuras (p.30).

Por otro lado, los aportes de la investigadora argentina Flavia Terigi (2007; 2009; 2010) ayudan a problematizar y diferenciar las trayectorias educativas teóricas, ideales, homogéneas de las trayectorias educativas reales, no encauzadas, heterogéneas.

Las primeras refieren a aquellos «recorridos de los sujetos en el sistema que siguen la progresión lineal prevista por este en los tiempos marcados por una periodización estándar» (Terigi, 2010, p.19) y suponen tres momentos del sistema educativo que las estructura: la organización del sistema por niveles, la gradualidad del currículum y la anualización de los grados de instrucción.

En cambio, las segundas se definen para referir a las trayectorias que efectivamente ocurren, es decir, las que no necesariamente y por diversos motivos, siguen el modelo educativo diseñado. Terigi (2010) las llama trayectorias no encauzadas «usando la metáfora de cauce de un río: se salen de cauce y [...] nos plantean algunos desafíos» (p.19).

Una problemática se ve en que las trayectorias teóricas estructuran el saber pedagógico y entonces el cómo enseñar depende del año académico en el que se encuentren las y los estudiantes pero a su vez responde al modelo construido sobre la expectativa que 
contiene la trayectoria teórica (Terigi, 2010). Por ende, es un desafío y un punto a trabajar tanto para las instituciones educativas como para el cuerpo docente, el ser capaces de articular propuestas pedagógicas que contemplen las heterogeneidades que convergen en el aula.

En el artículo Directores que Hacen Escuela de la OEI (2015) quiénes determinan a las trayectorias reales como «expresiones de un conjunto de condiciones socioeducativas diversas que atraviesan la vida escolar» (p.2). De este modelo, señalan una serie de rupturas y discontinuidades en este tipo de recorridos: ingreso tardío al sistema; abandono temporario; inasistencias reiteradas o prolongadas; repitencia; sobreedad; y rendimiento menor al esperado.

La configuración de las trayectorias estudiantiles está claramente condicionada por variables como su edad, su proveniencia, su trabajo, su familia, sus prácticas extracurriculares, sus hobbies, y, en estos dos años de cursadas virtuales, por su acceso real a dispositivos tecnológicos y de conectividad. Por ejemplo, hay estudiantes que tienen un trabajo remunerado, el cual les demanda una gran parte de su día y tienen que equilibrarlo con el tiempo que le dedican al estudio.

La pregunta por las tensiones existentes entre las actividades académicas y extraacadémicas que realizan las y los estudiantes, y el modo en que esto impacta y moldea su camino por la institución educativas constituye uno de los objetivos específicos que tiene este trabajo.

\section{Abordaje metodológico}

En este trabajo se asume una perspectiva metodológica cualitativa porque resulta la más adecuada, ya que propone una mirada interpretativa centrada en el entendimiento del significado de las acciones de los actores sociales (Hernández Sampieri, 2006), donde convergen varias realidades como la del propio investigador/a, la de las y los participantes y la que se produce en la interacción que se da entre estos actores sociales. 
Desde esta mirada metodológica no se busca investigar a alguien o a un grupo, sino que lo que se pretende es trabajar con alguien y con esos grupos, lo cual le permite a quien investiga posicionarse desde el campo y, en este caso, analizar las prácticas sociales desde una mirada comunicacional (Uranga et. al, 2009).

En cuanto a las herramientas metodológicas, de acuerdo al momento en que se sitúe el proceso de investigación, se trabajará con diferentes técnicas. Por un lado, con técnicas de registros para buscar, obtener, relevar información (como entrevista, el relevamiento documental y los grupos de enfoque). Por otro lado, se emplearán técnicas de análisis que me permitirán distinguir la información relevada en el campo para problematizarla, compararla y establecer categorías que aporten a la explicitación y apropiación del análisis.

Hasta el momento, se ha avanzado en la realización de entrevistas en profundidad con un universo de ocho estudiantes de la Licenciatura en Comunicación Social, que están cursando en los primeros tres años de la carrera. Algunas han podido ser presenciales, dado que residen en La Plata, y otras virtuales, desde la ciudad o pueblo natal donde el/la estudiante ha estado durante este contexto pandémico.

De este modo, es posible relevar algunos testimonios, que ilustran, a priori, de qué modo se están configurando sus trayectorias digitales en un contexto de virtualización de clases:

Para las cursadas uso a veces el celular o a veces la computadora, pasa que a la computadora se le rompió la cámara, entonces me conecto desde el celular. Prefiero imprimir pero es un costo, así que leo desde la compu. (Estudiante 01)

Toda la mañana, mientras curso uso la compu y trato de no usar el celular. Sigo leyendo, haciendo un trabajo básicamente con la compu o leyendo en papel para no interactuar con el celu y distraerme. (Estudiante 02)

[Me conecto por] el celular, y tenía una notebook buena pero ya no funciona. Una amiga de mi mamá nos prestó una notebook pero no es tan buena, así que solo la uso para hacer los trabajos. Por eso estoy haciendo videollamada por el celular. (Estudiante 03) 


\section{Desigualdades y diversidades en el acceso, uso y apropiación de las tecnologías}

El contexto de Aislamiento Social, Preventivo y Obligatorio (ASPO) (1) por COVID 19 obligó a las Universidades argentinas a la plena virtualización de clases en pos de seguir garantizando el derecho a la educación de todas y todos sus estudiantes. En efecto, este contexto de emergencia sanitaria develó y acentúo las persistentes condiciones de desigualdad en las que se encuentran las y los estudiantes universitarios/s respecto del acceso a dispositivos tecnológicos y de conectividad. Delia Crovi Druetta ha dedicado numerosos estudios al análisis de la brecha digital, tanto en Argentina como en América Latia, ya en 2013 afirmaba:

El concepto de brecha digital implicó reconocer que estamos ante un modelo social que genera exclusión y discriminación entre quienes no tienen acceso a los instrumentos que hacen posible tal modelo. Los jóvenes, como protagonistas del cambio, en mayor o en menor medida son víctimas de la brecha digital, tanto que buena parte de los programas diseñados para combatirlas están destinados a la juventud (2013, p.129)

Para complejizar la conceptualización, Crovi Drueta (2013) destaca que la brecha digital posee dos dimensiones: la tecnológica, vinculada al acceso a los ámbitos de la infraestructura tecnológica disponible y las condiciones materiales que hacen posible este acceso; y la cognoscitiva, diversa en el uso y se expresa a través del ejercicio de las habilidades informáticas y del capital cultural disponible en los usuarios/as.

Así, lo que denomina brecha cognitiva, es un aspecto resultante de la brecha digital que combina la falta de habilidades para manejar las innovaciones tecnológicas y la escasez del capital cultural de las y los jóvenes (Crovi Drueta, 2013). Por esta razón, afirma que para combatir la brecha digital hay que prestar atención al acceso a las TICs pero también a las habilidades desarrolladas para su uso y apropiación a la vez que considera que es urgente realizar estudios que den cuenta de los modos de uso

y apropiación cultural de las TICs. Esta es una de las preguntas a la que este proyecto busca aportar.

\footnotetext{
IICom (Instituto de Investigaciones en Comunicación)

Facultad de Periodismo y Comunicación Social

Universidad Nacional de La Plata
} 
Del mismo modo, Silvia Lago Martínez (2019) también se involucra en el debate en torno a las conceptualizaciones de la brecha digital dándole importancia a la cuestión de la apropiación cultural, planteando que «hoy en día es entendida como un concepto multidimensional que implica bastante más que el

acceso físico a las mismas, al tiempo que se incorpora la noción de apropiación tecnológica (párr.5) Particularmente, la apropiación de las tecnologías puede darse de diversas formas en los individuos o sujetos colectivos. Lago Martínez, Méndez y Gendler (2017) distinguen cuatro tipos o formas de hacerlo:

- Apropiación adaptativa o reproductiva, refiere a los aprendizajes, representaciones y valores en el uso de la tecnología de acuerdo con los fines para los que fue pensada. Aquí se concibe a la tecnología como instrumento o herramienta externa, el cual hay que "hacer propio".

- $\quad$ Apropiación adaptativa o creativa, contempla las formas nuevas de uso y aplicación de prácticas alternativas y originales de la tecnologías, como puede ser la creación de contenidos, aplicaciones o extensiones que permiten darle otros usos que difieren de las esperadas a priori.

- $\quad$ Apropiación cooptativa, tiene que ver con la apropiación de algo "externo" por parte de las corporaciones, los gobiernos y las empresas que son generalmente los creadores de artefactos, plataformas, software y hardware.

- $\quad$ Creación, esta última no lleva la denominación "apropiación" porque no busca incorporar a las tecnologías sino más bien pensar en el proceso de crear las propias tecnologías. Esto requiere de conocimientos saberes, estrategias y contextos que hagan posible el hecho de crear una tecnología única y distinta.

Vale preguntarse qué tipos de apropiación llevan adelante las y los estudiantes, específicamente los jóvenes comunicadores de la FPyCS-UNLP, ya que su carrera de grado y formación académica se ve absolutamente interpelada por la apropiación tecnológica. Por ejemplo, el trabajo de Porta, Urdaniz y Zapata (2018) se sitúa en el Proyecto de Investigación "Convergencia tecnológica y producción de sentidos: producción, edición, circulación y almacenamiento en red ¿otro/nuevo paradigma comunicacional? -donde se ubica esta beca- y brinda un claro ejemplo de experiencias educativas con tecnologías en el aula. 
En este caso, socializan los resultados del Trabajo Final Integrador (TFI) de la materia Modernidades, Medios y Poder (cát.II) donde las y los estudiantes deben elegir y analizar una narrativa audiovisual, articulando los contenidos vistos en este espacio curricular y presentando un producto transmedia como síntesis del mismo.

A partir del resultante de estas producciones, las autoras sostienen que la apropiación de las tecnologías «facilita el desarrollo de la propuesta de evaluación [...], vital para el proceso pedagógico si consideramos el típico temor que aparece en el estudiantado ante la situación de examen» (Porta, Urdaniz y Zapata, 2018).

Asimismo, ponen en discusión el rol de las tecnologías como transformadoras y generadoras de crisis en el lenguaje y en los modos de comunicar(se): La aceptación y uso de las tecnologías digitales móviles, mediante sus pequeñas pantallas de tres pulgadas, atrajo a millones de personas al mundo de la cultura de la virtualidad antes reservado a las elites y los sectores "educados", fomentando el uso de pantallas como lazos sociales, como espacios de diálogos que antes eran exclusivos del encuentro cara a cara (p.5)

Por tanto, proponen trabajar en el marco institucional y educativo el valor estratégico de la convergencia al tiempo que advierten una necesidad en comprender el contexto tecnocultural que se da en las instituciones educativas y los medios de comunicación y consideran que la intersección entre comunicación, educación y tecnologías es fundamental para analizar los modos de producir, almacenar y circular el conocimiento.

Por otro lado, el texto de Cuestas y Welschinger (2020) toma como eje la educación digital para reflexionar en torno a la virtualización de clases y el impacto de la pandemia por COVID-19 en la educación superior. Entonces plantean a la (des)conexión, a la digitalización como una nueva dimensión de la desigualdad: El tipo y la modalidad de acceso a la conectividad, el tipo de dispositivo tecnológico, las capacidades de gestión de la institución educativa para organizar y centralizar las propuestas pedagógicas de sus docentes, son todas variables que tienen un impacto disímil e las posibilidades de garantizar la continuidad pedagógica, en un sistema educativo marcado al extremo por la desigualdad (p.6). Además, hay que pensar en estrategias formativas no que busquen reemplazar las clases presenciales (ya que en el entender de este proyecto de beca, no lo 
son) sino que se puedan explotar las potencialidades y especificidades de estas nuevas mediaciones (Cuestas y Welschinger, 2020) por que sin una «apropiación activa, crítica y pedagógica» no hay recurso que valga.

Por último, recaen sobre la importancia del vínculo docente-estudiante para la continuidad pedagógica, dado que es absolutamente distinta la relación que puede establecer dentro del aula, cara a cara, que la que puede resultar a través de cuadrados en una plataforma de videollamada. «Interrumpir ese vínculo es potencialmente causa de abandono de la educación en todos sus niveles» (p.7).

Sin dudas, este es un escenario de grandes transformaciones frente al cual las instituciones, tanto la Universidad Nacional de La Plata como la Facultad de Periodismo y Comunicación Social tienen el gran desafío de dedicarle un tiempo de indagación, investigación y análisis para presentar propuestas que contemplen estas realidades digitales, la desigualdad presente y acentuada por la pandemia, y la diversidad en el uso y apropiación que tienen las y los estudiantes.

\section{Políticas universitarias para sostener las trayectorias}

Se parte de la concepción de la Universidad como derecho humano. Eduardo Rinesi (2014) piensa a la Universidad como el espacio en donde se hace efectivo el ejercicio del derecho que tienen todos y todas a la educación superior así como la herramienta primordial para la inclusión social y la ampliación ciudadana. El logro histórico de que el ingreso a las Universidades públicas argentinas sea irrestricto (2) implicó patear el tablero de la universidad formadora de élites, exclusivista y expulsante, y de este modo sí es posible hablar de «la democratización de la educación superior como garantía de la igualdad de oportunidades»(Juarros, 2006). Esto significa proyectar a la Universidad como igualadora, en contraposición del pensamiento neoliberal que sostiene a la meritocracia como premio al "esfuerzo" y modalidad de política educativa.

Retomando a Rinesi (2014), el concepto de democratización se define como proceso de universalización de una serie de derechos que son garantizados por el Estado, por lo que es 
este, y en su implicancia, la propia Universidad, la que debe hacer de este derecho un «proceso real, material y concreto» (p.10).

Sin embargo, con la garantización del acceso no alcanza. También hay que efectivizar la permanencia y el egreso de las y los estudiantes que llegan y transitan las universidades. La masificación de la educación universitaria supuso el ingreso masivo de miles de jóvenes y adultos/as que nunca habían pensado en la Universidad como un horizonte de posibilidad. Pero, ¿cuántos y cuántas de ellas permanecen adentro luego del primer año? ¿y qué número son los/as que efectivamente egresan?

De acuerdo a los datos relevados por la Universidad Nacional de La Plata por iniciativa de la Secretaría General de Presidencia, se identifica una evolución en cuanto a la cantidad de alumnos/as en todas las Unidades Académicas, siendo que en 2013 había un total de 96.440 y para 2019 este llegó a 114.119. Ahora bien, respecto de ingresantes, se incrementa de 21.374 a 30.187, pero tan solo 6338 obtuvieron sus títulos de grado en 2019 -versus 6094 de 2013-, lo cual es alarmante.

Fernando Tauber (2018), el actual presidente de la UNLP, se pronuncia respecto a la educación superior como un bien público y social, un derecho humano universal, y una responsabilidad del Estado. Asimismo, comenta la importancia de trabajar en políticas que atiendan las necesidades del estudiantado:

El mayor rendimiento académico y el incremento de la graduación en tiempos proporcionados con la vida útil de cada ciclo formativo son una condición imprescindible para situar a la universidad como una institución útil a la demanda insistente de un país y una sociedad que nos sostiene. Se debe atender las razones particulares y generar políticas para resolver las razones generales. No se puede dar el lujo como institución y como país, de tener un desgranamiento feroz, una duración laxa de las carreras ni de un raquítico nivel de egreso. No lo debemos permitir (p.10).

Entonces, la UNLP asume un compromiso pleno como la institución educativa pública, gratuita y de calidad que es, y emplea estrategias de acompañamiento académico y bienestar estudiantil. El trabajo de Ros, Benito, German y Justinaovich (2016) caracteriza al Programa de 
Sostenimiento a las Trayectorias Estudiantiles impulsado por la Secretaría Académica desde 2014 con el objetivo de comprender y reflexionar sobre la construcción de las trayectorias a la vez de identificar problemáticas y cuestiones institucionales que exceden al estudiantado.

A raíz del análisis de las trayectorias, las autoras pudieron identificar cuatros rasgos/dimensiones presentes en las mismas. Como primer rasgo, surge la discontinuidad relacionada a la baja intensidad con los dispositivos y estrategias institucionales; un segundo rasgo responde a la fragmentación entendida como un "bache" entre el tiempo de aprendizaje y el de la acreditación.

La tercera dimensión se relaciona al desfasaje entre los plazos de enseñanza y los de aprendizaje/comprensión de los conocimientos adquiridos en clase. Y por último, la cuarta refiere a la condición de recurrentes que tienen algunas trayectorias, es decir, de "recursada" en el caso de los y las estudiantes que evitan el examen final o desaprueban las instancias evaluativas previas.

Cheli, Montenegro y Marano (2020) dan cuenta de cada una de las políticas empleadas por la UNLP. Por un lado, se encuentran las estrategias de bienestar estudiantil que son «propuestas de índole socioeducativa, que posibilitan el acceso a bienes y servicios sociales y culturales que facilitan la continuidad de las trayectorias (p.7)». Por el otro lado, y en tanto estrategias académicas de apoyo al sostenimiento de trayectorias, se pueden mencionar las propuestas organizacionales, pedagógicas y curriculares.

\begin{tabular}{|c|c|}
\hline $\begin{array}{l}\text { Bienestar Estudiantil } \\
\text { (Presidencia de UNLP) }\end{array}$ & $\begin{array}{|llll|}\begin{array}{l}\text { Sostenimiento } \\
\text { Académica) }\end{array} & \text { de } & \text { Trayectorias } & \text { (Unidad } \\
\end{array}$ \\
\hline $\begin{array}{l}\text { - albergue y comedor } \\
\text { universitario; } \\
\text { - becas de estudio; } \\
\text { - becas de movilidad } \\
\text { (entrega de bicicletas); }\end{array}$ & $\begin{array}{l}\text { • Curso Introductorio o Cátedras de } \\
\text { Inicio; - Tutorías; } \\
\text { - Talleres de lectura, escritura y } \\
\text { oralidad; -Talleres o ciclo de charlas sobre } \\
\text { temáticas que amplíen la formación inicial; }\end{array}$ \\
\hline
\end{tabular}




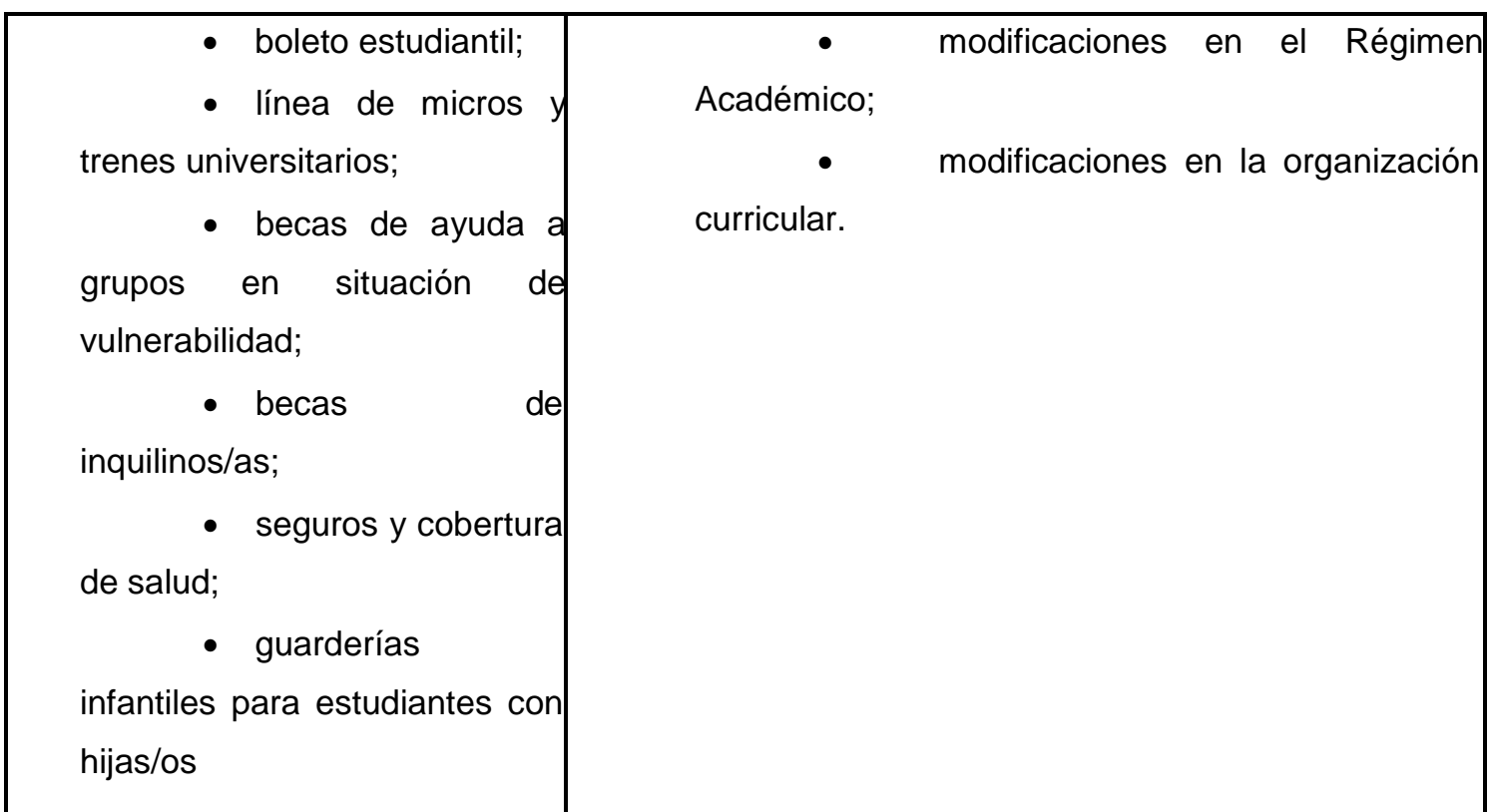

Tabla 1: Políticas universitarias de la UNLP.

Fuente: Elaboración propia, en base a lo relevado por Cheli, Montenegro y Marano (2020).

En mayo del 2020, un período atravesado por la pandemia de COVID-19 y consecuente virtualización de las clases, se diseñó e implementó la Beca "Tu PC para Estudiar" en el ámbito del Programa E-basura de la Secretaría de Vinculación e Innovación Tecnológica y la Dirección de Becas Universitarias de la Prosecretaría de Bienestar Universitario (perteneciente a Presidencia de UNLP) que tiene lugar en el programa de Igualdad de Oportunidades para Estudiar.

Esta beca está dirigida a aquellos y aquellas estudiantes que no tengan los recursos socioeconómicos y no cuenten con equipos para el efectivo acceso a internet, a quienes se les entregará una PC de escritorio, una Notebook o una Tablet durante el tiempo de cursada de sus carreras de grado. 
Además de las políticas universitarias ya mencionadas, desde la Facultad de Periodismo y Comunicación Social (FPyCS) se proponen muchas otras. La tesis de grado de Emilia Eizmendi, Francisco Idiart y Juliana Novello (2019) recupera el trabajo realizado por la gestión de esta Casa de Estudios durante 2010-2018, donde se lograron grandes avances en materia de inversión y ampliación

de derechos en la educación pública.

\begin{tabular}{|c|c|}
\hline & Políticas académicas inclusivas de la FPyCS \\
\hline Gremial & 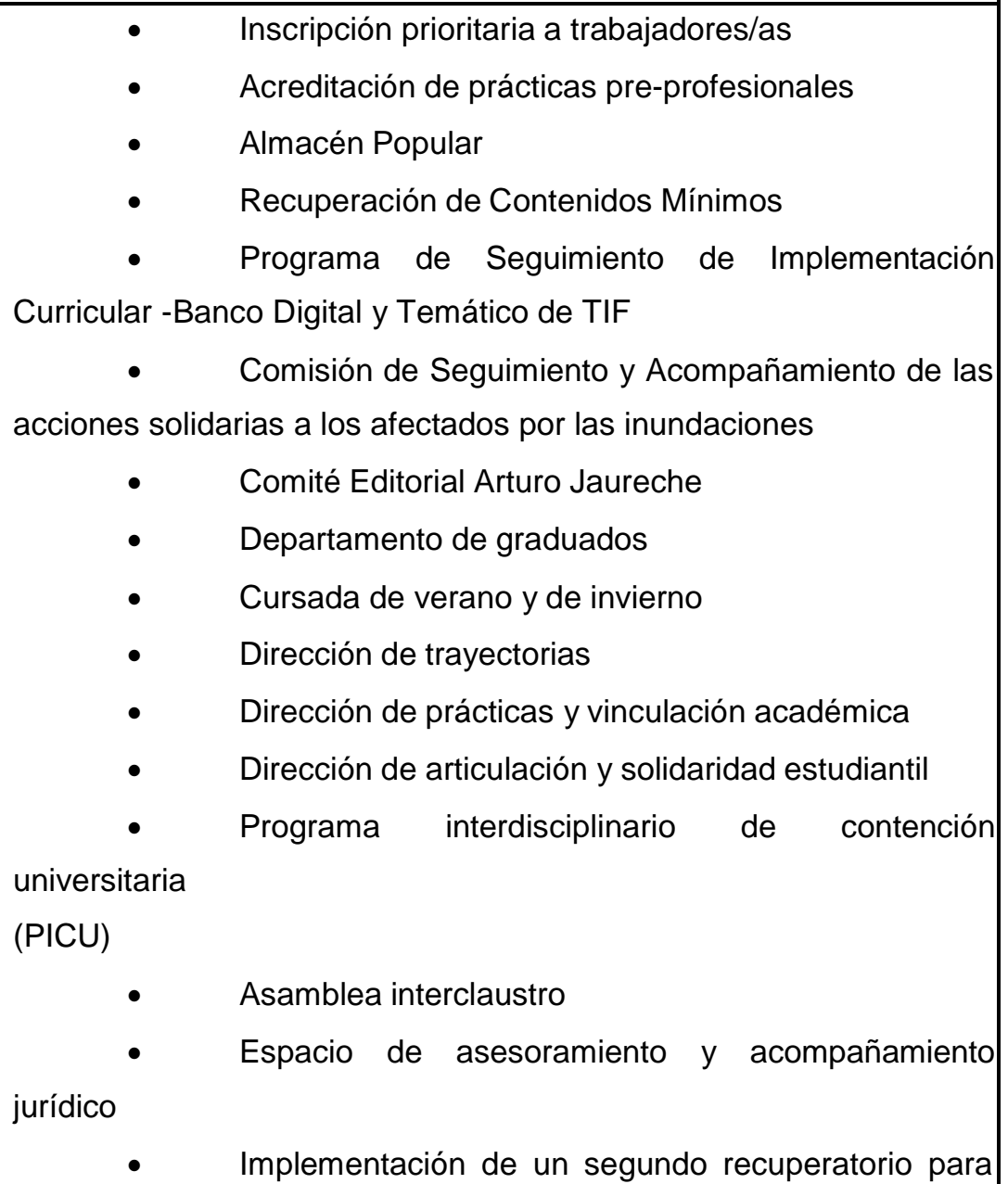 \\
\hline
\end{tabular}




\begin{tabular}{|c|c|c|}
\hline & \multicolumn{2}{|c|}{$\begin{array}{cl}\text { todas las carreras de grado } \\
\qquad \quad \text { Licencia por parentalidad } \\
\bullet & \text { Programa de tutorías } \\
\bullet & \text { Recibidas solidarias }\end{array}$} \\
\hline $\begin{array}{l}\text { Género(s) y } \\
\text { Diversidad(es) }\end{array}$ & de género & $\begin{array}{l}\text { Baños de uso común } \\
\text { Dirección de diversidad sexual } \\
\text { Secretaría de género } \\
\text { Espacio de intervención en casos de violencia } \\
\text { /G) }\end{array}$ \\
\hline $\begin{array}{l}\text { Comunicación } \\
\text { y Cultura }\end{array}$ & $\begin{array}{r}\bullet \\
\text { (Andén de } \\
\bullet \\
\bullet\end{array}$ & $\begin{array}{l}\text { Agencia de noticias deportivas nacionales } \\
\text { vo) } \\
\text { Semestres culturales } \\
\text { Hágalo circular } \\
\text { Lado B }\end{array}$ \\
\hline Extensión & $\begin{array}{r}\bullet \\
(\mathrm{CECAM}) \\
\bullet \\
\bullet \\
\bullet\end{array}$ & $\begin{array}{l}\text { Centro de Comunicación y Adultos Mayores } \\
\text { Extensiones áulicas } \\
\text { Takuru } \\
\text { Unidad de Prácticas (UdeP) }\end{array}$ \\
\hline Derechos & $\bullet$ & Dirección contra la Violencia Institucional \\
\hline
\end{tabular}




\begin{tabular}{|l|cc|}
\hline Humanos & $\begin{array}{c}\bullet \\
\bullet\end{array}$ & Dirección de Pueblos Originarios \\
opción de "capacitación de idioma" & Inclusión de idioma o lengua originaria como \\
de & & Programa de Educación Superior en Contexto \\
Encierro & \\
& - & Comisión de discapacidad \\
\hline
\end{tabular}

Tabla 2: Políticas académicas implementadas por la FPyCS para acompañar las trayectorias. Fuente: elaboración propia, de acuerdo a lo dispuesto por Eizmendi, Idiart y Novello (2019)

Como se pudo explicitar, la Facultad de Periodismo y Comunicación Social (FPyCSUNLP) tiene un claro objetivo de inclusión social como política universitaria y así se ve reflejado en estas estrategias de acompañamiento y sostenimiento a las trayectorias estudiantiles. Ahora queda preguntarse qué miradas, herramientas y estrategias hay específicamente para las trayectorias digitales, es decir, para afrontar las desigualdades en el acceso, uso y apropiación de dispositivos tecnológicos y de conectividad.

\section{Algunas visiones futuras}

Los estudios e investigaciones relevadas demuestran que las trayectorias educativas son experiencias diversas, heterogéneas, difusas y particulares en cada estudiante. De hecho, no hay un estudiante igual a otra/o y concebirse de manera cerrada y homogénea se vuelve un impedimento que no permite verlas/os como lo que son: sujetos interpeladas/os y atravesadas/os por su edad, su lugar de origen, su familia, sus sueños, su condición juvenil, sus hobbies, su trabajo, sus docentes, sus compañeras/as, su militancia.

La Universidad Nacional de La Plata y especialmente la Facultad de Periodismo y Comunicación Social son dos instituciones comprometidas con ampliar el universo de posibilidades de la ciudadanía y soñar con una Universidad más justa e inclusiva. Por ello, ya 
están empleando estrategias y herramientas que sirven de acompañamiento y sostenimiento de estas trayectorias para que ningún estudiante se quede afuera.

Atendiendo el contexto desigual, resulta imperante una mirada atenta y activa tanto institucional como pedagógica para con el acceso, uso y apropiación de las tecnologías en las y los estudiantes, teniendo en cuenta que los ciclos lectivos 2020 y 2021 han sido virtuales y por ende, el estudiantado sólo ha podido efectivamente acceder a las clases mediante plataformas que requieren de conectividad y un dispositivo a través del cual conectarse.

Es a partir de percibir un lugar de vacancia en la producción de conocimiento y sentidos respecto de las trayectorias estudiantiles digitales universitarias, que se busca indagar, ubicándose siempre desde la educación superior como un derecho humano y desde la necesidad de hacer completamente efectivo este derecho.

\section{Notas}

(1) Decreto 297/2020 de marzo 2020 que declaró el Aislamiento Social, Preventivo y Obligatorio en toda la Argentina.

https://www.boletinoficial.gob.ar/detalleAviso/primera/227042/20200320

(2) Las modificaciones a la Ley 24.521 de Educación Superior (LES), específicamente el artículo 7 dictamina: "Todas las personas que aprueben la educación secundaria pueden ingresar de manera libre e irrestricta a la enseñanza de grado en el nivel de educación superior". http://servicios.infoleg.gob.ar/infoleglnternet/anexos/250000-

254999/254825/norma.htm

\section{Referencias bibliográficas}

Bracchi, C. (2016). Descifrando el oficio de ser estudiantes universitarios: entre la desigualdad, la fragmentación y las trayectorias educativas diversificadas. Revista Trayectorias 
Universitarias,

2(3).

https://revistas.unlp.edu.ar/TrayectoriasUniversitarias/article/view/3019

Bourdieu, P. (1979). Los Tres Estados del Capital Cultural. Sociología, UAM- Azcapotzalco, México, núm 5, pp. 11-17.

Cheli, V., Montenegro, J., y Marano, M.G. (2020). "Clase 4: las trayectorias estudiantiles en la universidad". Universidad Nacional de La Plata. Programa de Capacitación Docente UNLP ADULP, 19 de junio.

Crovi Druetta, D. M. (2013). Jóvenes, migraciones digitales y brecha tecnológica. Revista Mexicana de Ciencias Políticas y Sociales, vol. LII, núm. 209. Recuperado de

https://www.redalyc.org/pdf/421/42116235008.pdf

Cuestas, P. y Welschinger, N. (2020). Apuntes para la política universitaria. Coronavirus: la educación (digital) interpelada. Instituto de Estudios y Capacitación (IEC) CONADU. Recuperado de https://iec.conadu.org.ar/files/publicaciones/1608679698_2020-coronavirus-laeducacion-digital-int erpelada.pdf

Directores que hacen Escuela. (2015). en colaboración con Joana López. De la trayectoria en singular a las trayectorias en plural. OEI, Buenos Aires. Recuperado de https://portaldelasescuelas.org/wp-content/uploads/2016/03/1_Trayectorias-

teoricas_y_reales.pdf 
Eizmendi, E., Idiart, F., y Novello, J. (2019). Políticas universitarias inclusivas: recopilación de las prácticas de gestión 2010-2018 en la FPyCS - UNLP. (Tesis de grado). Recuperado de http://sedici.unlp.edu.ar/handle/10915/96816

FILO UBA (5 de octubre de 2020). Desigualdad, capital cultural y trayectorias educativas en tiempos de pandemia [archivo de video]. Recuperado de https://www.youtube.com/watch?v=3NQbBw83FGw\&t=2420s

FPyCS-UNLP. (2014). Plan de Estudios Licenciatura en Comunicación Social, La Plata.

Hernández Sampieri, R. (2016). Et. al. Metodología de la investigación. México Distrito Federal, México: Mc Graw Hill.

Juarros, M.F. (2006). ¿Educación superior como derecho o como privilegio? Las políticas de admisión a la universidad en el contexto de los países de la región. Revista Andamios 3(5), Recuperado de https://www.redalyc.org/pdf/628/62830504.pdf

Lago Martínez, S. (Coord.). (2019). Políticas públicas e inclusión social. Recuperado de https://www.teseopress.com/politicaspublicaseinclusiondigital/front-matter/presentacion/

Lago Martínez, S., Méndez, A., y Gendler, M. (2017). Teorías, debates y nuevas perspectivas sobre la apropiación de tecnologías digitales (pp. 75-86). Contribuciones al estudio de procesos 
de apropiación de tecnologías. Ciudad Autónoma de Buenos Aires (Argentina): Ediciones del Gato Gris.

Porta, P., Urdaniz, A. y Zapata, N. (2018). Consumos culturales juveniles y apropiación de las plataformas digitales a partir de una producción narrativa transmedia en el aula. Conferencia en XXII Jornadas de la Red Nacional de Investigadores en Comunicación. Recuperado de http://sedici.unlp.edu.ar/bitstream/handle/10915/75191/Documento_completo.pdf?sequence=1\& is A llowed $=\mathrm{y}$

Rinesi, E. (2014). La Universidad como derecho. Revista Política Universitaria, 1(1).Instituto de Estudios y Capacitación (IEC) CONADU. Recuperado de http://biblioteca.clacso.edu.ar/Argentina/iec-conadu/20171114055305/pdf_844.pdf

Reguillo, R. (2013). Culturas juveniles. Formas políticas del desencanto. México: Siglo XXI Editores.

Ros, M., Benito, L., Germain, L., y Justianovich, S. (2016). Las trayectorias estudiantiles en la UNLP. Aportes para pensar los desafíos a las experiencias pedagógicas. Trayectorias Universitarias, 2(3). Recuperado de https://revistas.unlp.edu.ar/TrayectoriasUniversitarias/article/view/3022

Tauber, F. (2018). Pensar la Universidad. Proyecto institucional de la Universidad Nacional de La Plata 2018-2022. Universidad Nacional de La Plata, Argentina. Recuperado de http://sedici.unlp.edu.ar/handle/10915/68411 
Terigi, F (2007). "Los desafíos que plantean las trayectorias escolares". III Foro Latinoamericano de Educación: Jóvenes y docentes. La escuela secundaria en el mundo de hoy. Recuperado de

http://www.bibliopsi.org/docs/carreras/obligatorias/CFP/educacional/erausquin/Unidad\%204/Ed uca cional\%20Erausquin\%20-\%20\%20Terigi\%20\%20Los\%20desafios\%20que\%20plantean\%20las\%2 0trayectorias\%20escolares.pdf

(2014). Trayectorias escolares e inclusión educativa: del enfoque individual al desafío para las políticas educativas. Avances y desafíos de la educación inclusiva en Iberoamérica. Madrid, España: OEl.

Universidad Nacional de La Plata. (2020). La UNLP en números. Recuperado de https://unlp.edu.ar/indicadores/la-unlp-en-numeros-17580

Universidad Nacional de La Plata. (2020). Beca tu PC para Estudiar. Recuperado de https://unlp.edu.ar/estudiantes/beca-tu-pc-para-estudiar-17879

Uranga, Washington (2019). Et Al. El sentido de las técnicas en el diagnóstico desde la comunicación. Algunos ejemplos y propuestas para trabajar. (Documento de cátedra). Taller de planificación de procesos comunicacionales. Facultad de Periodismo y Comunicación Social. Universidad Nacional de La Plata, La Plata, Argentina. 